\title{
SHADOWS OF GRAPHICAL MEAN CURVATURE FLOW
}

\author{
WOLFGANG MAURER
}

\begin{abstract}
We consider mean curvature flow of an initial surface that is the graph of a function over some domain of definition in $\mathbb{R}^{n}$. If the graph is not complete then we impose a constant Dirichlet boundary condition at the boundary of the surface. We establish longtime-existence of the flow and investigate the projection of the flowing surface onto $\mathbb{R}^{n}$, the shadow of the flow. This moving shadow can be seen as a weak solution for mean curvature flow of hypersurfaces in $\mathbb{R}^{n}$ with a Dirichlet boundary condition.

Furthermore, we provide a lemma of independent interest to locally mollify the boundary of an intersection of two smooth open sets in a way that respects curvature conditions.
\end{abstract}

\section{INTRODUCTION}

A family $\left(M_{t}\right)_{t \in(0, T)}$ of hypersurfaces of $\mathbb{R}^{n+1}$ is said to move by mean curvature flow if there is a map $X: M \times(0, T) \rightarrow \mathbb{R}^{n+1}$ such that $X(\cdot, t)$ is an immersion for all $t \in(0, T)$ with $X(M, t)=M_{t}$ and $X$ solves

$$
\frac{d}{d t} X(p, t)=-H(p, t) \nu(p, t)
$$

where $M$ is a $n$-dimensional manifold, $H(\cdot, t)$ is the mean curvature of $M_{t}$ and $\nu(\cdot, t)$ its normal, such that $-H \nu$ is the mean curvature vector. If $M_{t}=\operatorname{graph} u(\cdot, t)$ for a family of functions $u(\cdot, t): \Omega \subset \mathbb{R}^{n} \rightarrow \mathbb{R}$, then $M_{t}$ moves by mean curvature flow if and only if $u$ solves the graphical mean curvature flow equation, which is the parabolic partial differential equation

$$
\frac{d}{d t} u=\sqrt{1+|D u|^{2}} \operatorname{div}\left(\frac{D u}{\sqrt{1+|D u|^{2}}}\right) .
$$

Graphical mean curvature flow was studied in 5 by Ecker and Huisken. They proved long-time existence for the mean curvature flow of entire graphs and showed that the solution stays graphical for all time. More recently, Sáez Trumper and Schnürer proved in [9] a long-time existence result for complete graphs. Starting from an open set $\Omega_{0}$ and a proper function $u_{0}: \Omega \rightarrow \mathbb{R}_{+}$, they showed the existence of a solution $u$ to graphical mean curvature flow with initial data $u_{0}$, where $u(\cdot, t)$ is defined on an open set $\Omega_{t}$ for $t \geq 0$. This solution will not develop singularities on a finite level but it can disappear to infinity forming a singularity at infinity-level. It was observed that the sets $\partial \Omega_{t}$ can be interpreted as a weak solution to mean curvature flow, starting from $\partial \Omega_{0}$, and that it coincides almost everywhere with the level-set flow as long as the latter does not fatten (Fig. 1). In [3] and [4 existence results analogous to that of Sáez and Schnürer are proven for some fully nonlinear flows of non-compact convex surfaces.

In this article we consider graphical mean curvature flow with a Dirichlet boundary condition. The existence result of this article reads as follows.

Theorem 1.1. Let $\Omega \subset \mathbb{R}^{n}$ be open, smooth, and mean convex, that is, its boundary $\partial \Omega \in C^{\infty}$ has nonnegative mean curvature $H[\partial \Omega] \geq 0$. Let $u_{0}: \bar{\Omega} \rightarrow[-\infty, \infty]$ be

2010 Mathematics Subject Classification. 53C44. 


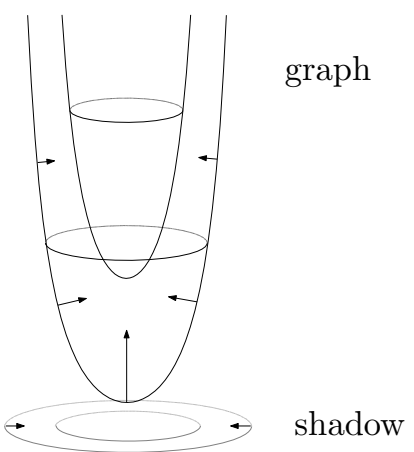

FiguRE 1. A rotationally symmetric "shadowflow" where we consider a graph over a ball. The graph moves by mean curvature flow and the shadow evolves by mean curvature flow too, but in a weak sense. In this setting the graph will disappear to infinity in finite time, while the shadow develops a point singularity. Generally, any singularity occuring on the shadow-level will happen at infinity on the graph-level.

continuous and assume $u_{0}$ is locally Lipschitz in the set $\left\{x \in \bar{\Omega}:\left|u_{0}(x)\right|<\infty\right\}$ and $\left.u_{0}\right|_{\partial \Omega}$ is of class $C^{2}$ in $\left\{x \in \partial \Omega:\left|u_{0}(x)\right|<\infty\right\}$. Then there is a continuous function $u: \bar{\Omega} \times[0, \infty) \rightarrow[-\infty, \infty]$ which is smooth on $(\Omega \times(0, \infty)) \cap\{|u|<\infty\}$ and solves

$$
\begin{cases}\dot{u}=\sqrt{1+|D u|^{2}} \operatorname{div}\left(\frac{D u}{\sqrt{1+|D u|^{2}}}\right) & \text { in }(\Omega \times(0, \infty)) \cap\{|u|<\infty\} \\ u(x, t)=u_{0}(x) & \text { for }(x, t) \in \mathcal{P}(\Omega \times(0, \infty))\end{cases}
$$

where $\mathcal{P}(\Omega \times(0, \infty)):=(\Omega \times\{0\}) \cup(\partial \Omega \times[0, \infty))$.

The condition of nonnegative mean curvature for the boundary $\partial \Omega$ is necessary to expect longtime existence for the graphical mean curvature flow when considering the Dirichlet problem for general boundary data. Otherwise, the gradient of $u$ may become unbounded in finite time and the flowing surface may cease to be graphical.

Note that for $\Omega=\mathbb{R}^{n}$ Theorem 1.1 is a generalization of the result in [9] because we do not need the assumptions $u_{0}$ proper and $u_{0} \geq 0$.

The proof of Theorem 1.1 is the subject of Section 2, It involves an approximation of the problem by bounded auxiliary problems and uses an Arzelà-Ascoli-argument and a priori estimates to pass to a limit.

In Section 3, we define the "shadow" at time $t$ to be the set

$$
\left\{x \in \mathbb{R}^{n}:|u(x, t)|<\infty\right\},
$$

and interpret this as a weak solution to mean curvature flow of hypersurfaces in $\Omega$ with Dirichlet boundary condition on $\partial \Omega$ using the notion of avoidance principle as defined in Section 3. Roughly, the definition of weak solution implies that any classical solution starting inside the shadow stays inside and any classical solution starting outside stays outside.

An intersection of two smooth open sets is in general not smooth at the intersection of the boundaries. In Section 4 we provide a lemma to locally mollify intersections of smooth open sets at the intersections of their boundaries while preserving certain curvature conditions. 
Theorem 1.2. Let $A, B \subset \mathbb{R}^{n}$ be open, $\partial A, \partial B \in C^{\infty}$ and suppose $A \cap B \neq \emptyset$ is bounded. Then for any $\varepsilon>0$ there is an open set $\Omega$ with $\partial \Omega \in C^{\infty}$ such that

$$
(A \cap B) \backslash(\partial A \cap \partial B)_{\varepsilon} \subset \Omega \subset A \cap B,
$$

where $(\partial A \cap \partial B)_{\varepsilon}:=\left\{x \in \mathbb{R}^{n}: \operatorname{dist}(x, \partial A \cap \partial B)<\varepsilon\right\}$.

Moreover, if the principal curvatures at every point of $\partial A$ and $\partial B$ lie in a symmetric, open or closed, convex cone $\Gamma \subset \mathbb{R}^{n-1}$ which contains the positive cone $\Gamma_{+} \subset \Gamma$, then $\Omega$ can be chosen such that the principal curvatures at any point of $\partial \Omega$ lie in $\Gamma$, too. Here symmetric means invariant when interchanging $\kappa_{i} \leftrightarrow \kappa_{j}$.

Example 1.3. Choosing $\Gamma=\left\{\left(\kappa_{1}, \ldots, \kappa_{n-1}\right): \kappa_{i} \geq 0\right.$ for all $\left.i\right\}$ corresponds to convex subsets. Choosing $\Gamma=\left\{\left(\kappa_{1}, \ldots, \kappa_{n-1}\right): \sum \kappa_{i} \geq 0\right\}$ corresponds to meanconvex domains. This is what we use in Section 2 ,

The problem of mollifying inside convex curvature cones was an open problem from the problem section of the conference Geometric evolution equations which took place in Konstanz in 2011. The proof uses distance functions to the boundaries and a mollified version of the minimum of these. It can be read independently of the other sections and is applied most noteably in Section 2 in the approximation process. The author expects that this result is of great use and will be widely applicable.

This article emerged from the author's master thesis and is meant to gather the main results. The author wishes to thank Oliver Schnürer for supervising the thesis and for his great support. The author also likes to thank Ben Lambert for helpful advice.

\section{Existence}

We prove the existence result Theorem 1.1 by approximating by auxiliary problems and using a priori estimates.

Proof of Theorem 1.1. We cut off the initial function $u_{0}$ in height by considering

$$
\bar{u}_{0, R}: \widetilde{\max }\left(\widetilde{\min }\left(u_{0}, R\right),-R\right)
$$

for $R>0$, where $\widetilde{\max }$ and $\widetilde{\min }$ are mollified versions of max and min respectively (defined analogously to 4.1 setting $\delta=1 / 2$ there). Next, we cut off the domain of definition $\Omega$ by intersecting with a ball $B_{2 R} \equiv B_{2 R}(0)$ and using Theorem 1.2 . This gives smooth open sets $\Omega \cap B_{R} \subset \Omega_{R} \subset \Omega \cap B_{2 R}$ whose boundaries have nonnegative mean curvature $H\left[\partial \Omega_{R}\right] \geq 0$. Finally, we restrict the functions $\bar{u}_{0, R}$ to $\Omega_{R}$ and take a mollification to find smooth functions $u_{0, R}$ defined on $\Omega_{R}$ satisfying $\left\|u_{0, R}-\bar{u}_{0, R}\right\|_{L^{\infty}\left(\Omega_{R}\right)}<R^{-1}$ and $\left\|u_{0, R}-\bar{u}_{0, R}\right\|_{C^{2}\left(\partial \Omega \cap B_{R}\right)}<R^{-1}$.

Now define $u_{R}$ as the solution of the auxiliary problem

$$
\begin{cases}\dot{u}_{R}=\sqrt{1+\left|D u_{R}\right|^{2}} \operatorname{div}\left(\frac{D u_{R}}{\sqrt{1+\left|D u_{R}\right|^{2}}}\right) & \text { in } \Omega_{R} \times(0, \infty), \\ u_{R}(x, t)=u_{0, R}(x) & \text { for }(x, t) \in \mathcal{P}\left(\Omega_{R} \times(0, \infty)\right) .\end{cases}
$$

By [7, Theorem 2.1], $u_{R}$ is well-defined and satisfies

$$
\begin{array}{ll} 
& u_{R} \in C^{0}\left(\overline{\Omega_{R}} \times[0, \infty)\right) \cap C^{\infty}\left(\Omega_{R} \times(0, \infty)\right) \\
\text { and } \quad D u_{R} & \in C^{0}\left(\overline{\Omega_{R}} \times[0, \infty)\right) .
\end{array}
$$

(By de Giorgi-Nash-Moser-estimates the spatial derivative $D u_{R}$ is even Höldercontinuous for some exponent.)

To be able to utilize the Arzelà-Ascoli-Theorem and to pass to a limit and obtain a solution of the initial problem, we are going to need local a priori estimates. These 
estimates will be local in space, time, and height: Due to the unboundedness in height we can only expect estimates at points $(x, t)$ depending on $\left|u_{R}(x, t)\right|$.

Since by Lemma 2.1 and Lemma 2.2 we have local gradient bounds at the boundary, local gradient estimates easily follow from the results of Section 2 in [5]. Using spheres as barriers we obtain Hölder-estimates in time with exponent $1 / 2$ (cf. Section 6 of [9]). This is sufficient to apply an Arzelà-Ascoli argument.

To use Arzelà-Ascoli for unbounded functions, simply compose with a homeomorphism $\Phi:[-\infty, \infty] \rightarrow[-1,1]$ which is smooth on $(-\infty, \infty)$. Then the gradient and Hölder-in-time estimates give locally uniform estimates for $\Phi \circ u_{R}$ where $\Phi \circ u_{R} \in(-1+\varepsilon, 1-\varepsilon)$, which is sufficient: Locally in space-time there is for any $\varepsilon>0$ a $\delta>0$ such that for almost all $R \in \mathbb{N}$ we have

$$
|(x, t)-(y, s)|<\delta \Rightarrow\left|\Phi \circ u_{R}(x, t)-\Phi \circ u_{R}(y, s)\right|<\varepsilon .
$$

By Arzelà-Ascoli a subsequence of $\left(\Phi \circ u_{R}\right)_{R \in \mathbb{N}}$ converges locally uniformly to a continuous function on $\bar{\Omega} \times[0, \infty)$ as $R \rightarrow \infty$. This correspondes to pointwise convergence of a subsequence of $\left(u_{R}\right)$ to a continuous function $u: \bar{\Omega} \times[0, \infty) \rightarrow$ $[-\infty, \infty]$ and locally uniform convergence on $\bar{\Omega} \times[0, \infty) \cap\{|u|<\infty\}$.

Then, using the interior estimates for higher derivatives in [5], one has locally smooth convergence on $\Omega \times(0, \infty) \cap\{|u|<\infty\}$ and we see that $u$ solves the same differential equation there as the $u_{R}$, which completes the proof.

Now we are going to establish the a priori estimates. Most importantly we need

Lemma 2.1 (local gradient estimates at the boundary). Let $\Omega$ be as in the statement of Theorem 1.1. $x_{0} \in \partial \Omega, r, T>0, B_{r}:=B_{r}\left(x_{0}\right) \cap \Omega$ and $\Gamma_{r}:=\partial \Omega \cap B_{r}\left(x_{0}\right)$. Let $u \in C^{2 ; 1}\left(B_{2 r} \times(0, T)\right) \cap C^{0}\left(\overline{B_{2 r}} \times[0, T]\right)$ be a solution of

$$
\begin{cases}\dot{u}=\sqrt{1+|D u|^{2}} \operatorname{div}\left(\frac{D u}{\sqrt{1+|D u|^{2}}}\right) & \text { in } B_{2 r} \times(0, T), \\ u(x, 0)=u_{0}(x) & \text { for } x \in B_{2 r}, \\ u(x, t)=\varphi(x) & \text { for } x \in \Gamma_{2 r}, t>0 .\end{cases}
$$

For the initial- and boundary data assume $u_{0} \in \operatorname{Lip}\left(\overline{B_{2 r}}\right)$ and $\varphi \in C^{2}\left(\overline{B_{2 r}}\right)$. Assume $D u \in C^{0}\left(\overline{B_{2 r}} \times[0, T]\right)$. Then on $\Gamma_{r} \times[0, T]$ we have

$$
|D u| \leq C\left(n,\|u\|_{L^{\infty}\left(B_{2 r} \times(0, T)\right)},\left\|u_{0}\right\|_{\operatorname{Lip}\left(B_{2 r}\right)},\|\varphi\|_{C^{2}\left(\overline{B_{2 r}}\right)}, r, \Gamma_{3 r}\right) .
$$

Proof. A nonlocal version of this result can be found, for example, in [6, Chapter 1.4]. There, one uses the barriers

$$
w^{ \pm}:=\varphi \pm \delta \log (1+\sigma d),
$$

where $d$ is the signed distance function to $\partial \Omega$ and the constants are chosen like $\delta \ll 1 \ll \sigma(\delta)$. This barrier works on an $\varepsilon$-neighbourhood of $\partial \Omega$, where $\varepsilon$ is chosen as $\sigma^{-1 / 2}$ and so small, that we are in a tubular neighbourhood. Because of $w^{ \pm}=\varphi$ on the boundary we find the desired gradient bound on the boundary by the comparison principle for Dirichlet boundary conditions.

To obtain a local version simply add $\pm\left(\|u\|_{L^{\infty}}+\|\varphi\|_{L^{\infty}}\right) \cdot \eta$ to the barriers, where $\eta \in C^{\infty}\left(\overline{B_{2 r}}\right)$ satisfies $0 \leq \eta \leq 1, \eta \equiv 0$ on $B_{r}$ and $\eta \equiv 1$ on $B_{2 r} \backslash B_{3 r / 2}$. The proof then works as in the nonlocal case but the choice of $\delta$ and $\sigma$ depends additionally on $r$ because the bounds on derivatives of $\eta$ depend on $r$.

To apply Lemma 2.1 we need local $L^{\infty}$-estimates on $u$. For this purpose we have the following

Lemma 2.2 ( $L^{\infty}$-estimates near the boundary). Let $\Omega$ be as before, $R, T>0$ and $x_{0}, B_{R}$ and $\Gamma_{R}$ defined as in Lemma 2.1. Let $u \in C^{2 ; 1}\left(B_{R} \times(0, T)\right) \cap C^{0}\left(\overline{B_{R}} \times[0, T]\right)$ 
be a solution of $\mathrm{GMCF}$ on $B_{R} \times(0, T)$. Then there exists $r>0$ dependent on $B_{R}, \Gamma_{R}$ and $T$, such that

$$
\sup _{B_{r} \times(0, T)}|u| \leq C,
$$

where $C>0$ depends on the same quantities as $r$ and additionally depends on $\sup _{\left(B_{R} \times\{0\}\right) \cup\left(\Gamma_{R} \times(0, T)\right)}|u|$.

Proof. Without loss of generality we may assume $x_{0}=0$ and that the inner normal to $\partial \Omega$ at $x_{0}$ is $e_{n}=(0, \ldots, 0,1)$. We are going to write the boundary locally as a graph: There is an open ball $B$ in $\mathbb{R}^{n-1}$ with center at the origin and $s>0$, such that

$$
B_{R} \cap(B \times(-s, s))=\left\{\left(\hat{x}, x^{n}\right) \in B \times(-s, s): h(\hat{x})<x^{n}\right\}
$$

and $\Gamma_{R} \cap(B \times(-s, s))=$ graph $h$ for some function $h \in C^{\infty}(\bar{B})$. Because of $H[\partial \Omega] \geq 0, h$ satisfies the differential inequality

$$
\operatorname{div}\left(\frac{D h}{\sqrt{1+|D h|^{2}}}\right) \geq 0 \text {. }
$$

Take $0 \leq \eta \in C_{0}^{\infty}(B), \eta \neq 0$, such that $v_{0}:=h+\eta$ fulfils $\left|v_{0}\right|<s$. Finally, define $v \in C^{\infty}(B \times(-1, T+1)) \cap C^{0}(\bar{B} \times[-1, T+1])$ to be the solution of

$$
\begin{cases}\dot{v}=\sqrt{1+|D v|^{2}} \operatorname{div}\left(\frac{D v}{\sqrt{1+|D v|^{2}}}\right) & \text { in } B \times(-1, T+1), \\ v(\hat{x}, t)=v_{0}(\hat{x}) & \text { for }(\hat{x}, t) \in \mathcal{P}(B \times(-1, T+1)) .\end{cases}
$$

This way we have $v \in C^{\infty}(\bar{B} \times[0, T])$. By the maximum principle we also have $|v|<s$, and by the strong maximum principle $h(\hat{x})<v(\hat{x}, t)$ holds for all $x \in B$ and $t>-1$.

Define $Q$ by

$$
Q:=\left\{\left(\hat{x}, x^{n}, t\right) \in B \times(-s, s) \times(0, T): h(\hat{x})<x^{n}<v(\hat{x}, t)\right\},
$$

and choose $r>0$ from our assertion such that $B_{r} \times(0, T) \subset Q$ and $B_{r} \times(0, T)$ has positive distance to graph $v$.

Now we construct a barrier on $Q$ with the aid of the function $v$. On $Q$ define

$$
w(x, t):=\left(v(\hat{x}, t)-x^{n}\right)^{-1} \equiv\left(v(x, t)-x^{n}\right)^{-1},
$$

setting $v(x, t) \equiv v(\hat{x}, t)$ for $(x, t) \in Q$. One easily verifies that the level-sets of $w$ move by mean curvature, so that $w$ solves

$$
\dot{w}-\left(\delta^{i j}-\frac{w^{i} w^{j}}{|D w|^{2}}\right) w_{i j}=0
$$

Using this fact we calculate

$$
\begin{aligned}
\dot{w}- & \sqrt{1+|D w|^{2}} \operatorname{div}\left(\frac{D w}{\sqrt{1+|D w|^{2}}}\right)=\dot{w}-\left(\delta^{i j}-\frac{w^{i} w^{j}}{1+|D w|^{2}}\right) w_{i j} \\
& =-\frac{w^{i} w^{j}}{|D w|^{2}\left(1+|D w|^{2}\right)} w_{i j} \\
& =\frac{1}{1+|D w|^{2}}\left(\frac{v_{i j} w^{i} w^{j}}{\left(v-x^{n}\right)^{2}|D w|^{2}}-2|D w|^{2}\left(v-x^{n}\right)\right) \\
& \geq-\frac{\left\|D^{2} v\right\|_{L^{\infty}(Q)}}{|D w|^{2}\left(v-x^{n}\right)^{2}}-2\left(v-x^{n}\right) \geq-4 s^{2}\left\|D^{2} v\right\|_{L^{\infty}(Q)}-4 s \\
& \geq-c
\end{aligned}
$$


where $c>0$ is a constant that ultimately only depends on $B_{R}, \Gamma_{R}$ and $T$ through the above construction. Finally,

$$
w^{ \pm}:= \pm\left(w+c t+\sup _{\left(B_{R} \times\{0\}\right) \cup\left(\Gamma_{R} \times(0, T)\right)}|u|\right)
$$

are upper and lower barriers for $u$ on $Q$ respectively. Observe

$$
w(y, t) \rightarrow \infty \text { for } y \rightarrow x \in \mathcal{P}(Q) \backslash\left[\left(B_{R} \times\{0\}\right) \cup\left(\Gamma_{R} \times(0, T)\right)\right] .
$$

Now, on $B_{r} \times[0, T], w^{ \pm}$and therefore $|u|$ are bounded by a constant as in the assertion.

Remark 2.3. It is worth pointing out the solution is not unique in general: Consider two so called grim reaper curves lying next to each other, i. e. the graph of the function

$$
u_{0}(x):=-\log |\sin x| \quad \text { for } 0 \neq x \in(-\pi, \pi) .
$$

We can write down a translating solution to $\mathrm{GMCF}$ with initial data $u_{0}$ :

$$
\hat{u}(x, t):=t-\log |\sin x| \quad \text { for } 0 \neq x \in(-\pi, \pi), t \in \mathbb{R} .
$$

But the solution $u$ we have constructed in the proof of Theorem 1.1 differs from this translating solution $\hat{u}$ : The two grim reapers get connected at infinity. This is because we cut off the function $u_{0}$ at some height $R$ in the approximation process. To see that the approximating solutions $u_{R}$ do not converge to $\hat{u}$ we may consider the integral of the difference:

$$
\frac{d}{d t} \int_{-\pi}^{\pi} \hat{u}(\cdot, t)-u_{R}(\cdot, t)=\int_{\operatorname{graph} \hat{u}(\cdot, t)} \hat{K}-\int_{\operatorname{graph} u_{R}(\cdot, t)} K_{R} \geq 2 \pi-\pi=\pi,
$$

where $\hat{K}, K_{R}$ are the respective curvatures. By the maximum principle the convergence $u_{R} \rightarrow u$ is monotone and thus $\int u_{R}(\cdot, t) \rightarrow \int u(\cdot, t)$ as $R \uparrow \infty$. We conclude $\int(\hat{u}-u)(\cdot, t) \geq \pi t$ and therefore $u \neq \hat{u}$.

\section{The SHADOW-FLOW}

In this section we are going to investigate the projections/shadows of graphical mean curvature flow, that is the sets $\{|u|<\infty\}$ where $u$ is as in Theorem 1.1. We show that this shadow is a weak solution of mean curvature flow, where we use the following notion of weak solution which is based on the avoidance principle.

For this section we do not need to assume constant Dirichlet boundary values.

Let $\Omega$ be as in the last section.

Definition 3.1 (Weak solutions). A family $\left(A_{t}\right)_{t \in[0, \infty)}$ of open subsets of $\bar{\Omega}$ is called

- a supersolution to mean curvature flow if the following holds: For any family $\left(B_{t}\right)_{t \in[a, b]}$ of open sets, such that $B_{t} \Subset \Omega$ with $\partial B_{t} \in C^{\infty}$, and such that $\left(\partial B_{t}\right)_{t \in[a, b]}$ is a classical solution to mean curvature flow, we have

$$
\overline{B_{a}} \subset A_{a} \Rightarrow B_{b} \subset A_{b} .
$$

- a subsolution to mean curvature flow, if $\left(\bar{\Omega} \backslash \overline{A_{t}}\right)_{t \in[0, \infty)}$ is a supersolution.

- a weak solution of mean curvature flow, if $\left(A_{t}\right)_{t \in[0, \infty)}$ is both a super- and subsolution.

We shall call $\left(\partial \Omega \cap A_{t}\right)_{t \in[0, \infty)}$ the boundary values of $\left(A_{t}\right)_{t \in[0, \infty)}$. 
Remark 3.2. In the above setting in the definition of supersolution we even have $\overline{B_{t}} \subset A_{t}$ for all $t \in[a, b]$, when $\left(A_{t}\right)_{t}$ is a supersolution. To see that the closure is contained one can use, for instance, the translation invariance of classical flows.

If $\left(A_{t}\right)$ is a family of open subsets of $\bar{\Omega}$ such that $\left(\partial A_{t}\right)$ is a classical solution to mean curvature flow, then, by the avoidance principle, $\left(A_{t}\right)$ is a weak solution.

Let $x \in \partial A_{t} \cap \Omega$ and suppose that $\partial A_{t}$ is smooth in a ball $B_{r}(x) \subset \mathbb{R}^{n}$. If $\left(A_{t}\right)$ is a weak solution and $\partial A_{t}$ is smooth in a spacetime-neighbourhood of $(x, t)$, then $\left(\partial A_{t}\right)$ solves mean curvature flow at $(x, t)$ classically.

Proof. We may assume, that $\partial A_{t} \cap B_{r}(x)$ is the graph of a smooth function. Using the result of the next section we find two smooth open sets, one lying inside $A_{t} \cap B_{r}$, the other inside $B_{r}(x) \backslash A_{t}$, and such that their boundaries coincide in a neighbourhood of $x$ with $\partial A_{t}$. Taking $r$ to be small enough, small translations of these two open sets away from $\partial A_{t}$ serve as barriers. This way it can be seen, that the normal velocity of $\partial A_{t}$ at $(x, t)$ coincides with the mean curvature at that point.

Similar weak notions of mean curvature flow are the set-theoretic subsolutions of Ilmanen ([8]) or more generally the barriers of De Giorgi. Both of them were compared to the level-set flow (see [1] for a comparison of De Giorgi's barriers to level-set flow). (See [10] for a definition of set-theoretic subsolutions including boundary values.)

Note that our definition of weak solutions is not very useful where $\partial A_{t} \subset \partial \Omega$ (taking the boundary $\partial A_{t}$ relative to $\bar{\Omega}$ ). This is because there is no space left for a classical solution, that could possibly push $\partial A_{t}$ inwards into $\Omega$. To circumvent this, one could compare with classical solutions with boundary values that may not be written as the boundary of an open set and which can intersect $\partial A_{t}$. But this would cause trouble in the methods we are going to use next. Another way would be to compare $\partial A_{t}$ not only with classical solutions in $\Omega$ but with classical solutions that are boundaries of open subsets in $\mathbb{R}^{n}$ and which do not intersect the boundary values. This viewpoint has the disadvantage of not being intrinsically in $\bar{\Omega}$. The methods presented in the following also work if one adopts this definition for weak solutions.

Proposition 3.3. For any open set $A \subset \Omega$ there exists a weak solution of mean curvature flow $\left(A_{t}\right)_{t \in[0, \infty)}$ with $A_{0}=A$. Furthermore there is a smallest such weak solution $\left(A_{t}\right)_{t \in[0, \infty)}$ : For any weak solution $\left(A_{t}^{\prime}\right)_{t \in[0, \infty)}$ with $A \subset A_{0}^{\prime}$ we have $A_{t} \subset A_{t}^{\prime}$ for all $t \in[0, \infty)$.

Proof. Let $\mathcal{B}_{0}$ be the set of all families $\left(B_{t}\right)_{t \in[0, b]}$ of open sets such that $\overline{B_{0}} \subset A$ and $\partial B_{t} \in C^{\infty}$ fulfils mean curvature flow. Define

$$
A_{t}^{(0)}:=\bigcup\left\{B_{t}:\left(B_{t^{\prime}}\right)_{t^{\prime} \in[0, b]} \in \mathcal{B}_{0} \text { with } t \in[0, b]\right\} .
$$

Then inductively define $\mathcal{B}_{k}$ and $A^{(k)}$ by setting $\mathcal{B}_{k}$ to be the set of all families $\left(B_{t}\right)_{t \in[a, b]}$ of open sets such that $\overline{B_{a}} \subset A_{a}^{(k-1)}$ and $\partial B_{t} \in C^{\infty}$ fulfils mean curvature flow. Then set

$$
A_{t}^{(k)}:=\bigcup\left\{B_{t}:\left(B_{t^{\prime}}\right)_{t^{\prime} \in[a, b]} \in \mathcal{B}_{k} \text { with } t \in[a, b]\right\} .
$$

Finally define the open sets $A_{t}:=\bigcup_{k \in \mathbb{N}} A_{t}^{(k)}$. Note that $A_{t}^{(k)}$ is a nondecreasing sequence of open sets. As a union of subsolutions $\left(A_{t}\right)$ is again a subsolution. To see that $\left(A_{t}\right)$ is a supersolution let $\left(B_{t}\right)_{t \in[a, b]}$ be a classical solution and let $\overline{B_{a}} \subset A_{a}$. Then by compactness $\overline{B_{a}} \subset A_{a}^{(k)}$ for some $k \in \mathbb{N}$. Therefore $\overline{B_{b}} \subset A_{b}^{(k+1)} \subset A_{b}$ and hence $\left(A_{t}\right)$ is a supersolution.

The second assertion is obvious from the construction. 
The following lemma concerning weak solutions will be useful. The result is trivial for classical solutions.

Lemma 3.4. Suppose $\left(A_{t}\right)_{t \in[0, \infty)}$ is a family of open subsets of $\bar{\Omega}$, such that $\left(A_{t} \times\right.$ $\mathbb{R})_{t}$ is a weak solution of mean curvature flow in $\bar{\Omega} \times \mathbb{R}$.

Then $\left(A_{t}\right)_{t}$ is a weak solution of mean curvature flow in $\bar{\Omega}$.

Proof. We only show, that $\left(A_{t}\right)$ is a supersolution using the fact that $\left(A_{t} \times \mathbb{R}\right)$ is a supersolution.

Let $\left(B_{t}\right)_{t \in[a, b]}$ be a family of open sets such that $\partial B_{t} \in C^{\infty}$ fulfils mean curvature flow, and $\overline{B_{a}} \subset A_{a}$. We need to show $B_{b} \subset A_{b}$. In fact we prove $B_{b} \times \mathbb{R} \subset A_{b} \times \mathbb{R}$. For this we approximate $B_{a} \times \mathbb{R}$ by bounded sets.

For $R>0$ take $\hat{K}^{R}$ to be a smoothed intersection of $B_{a} \times \mathbb{R}$ with $\left\{\left|x^{n+1}\right|<2 R\right\}$ containing $B_{a} \times[-R, R]$ (use Theorem 1.2). We may take the same closing ends for different $R>1$, to give curvature bounds on $\partial \hat{K}^{R}$ independent of $R$. Then define $K^{R}:=\left\{x \in \hat{K}^{R}: \operatorname{dist}\left(x, \partial \hat{K}^{R}\right)>R^{-1}\right\}$. Then we still have uniform curvature bounds for $R>R_{0}$ sufficiently large. Thus, by Proposition 4.1 of 5 there are classical solutions $\left(M_{t}^{R}\right)_{t \in[a, a+\tau]}$ of mean curvature flow with $M_{a}^{R}=\partial K^{R}$ for some $\tau>0$ independent of $R$. These solutions $\left(M_{t}^{R}\right)$ are written as graphs over the $\partial \hat{K}^{R}$, which contain $\partial B_{a} \times[-R, R]$.

By interior estimates of 5 and the uniqueness of the limit (see below) we find for $R \rightarrow \infty$ local convergence as graphs over $\partial B_{a} \times \mathbb{R}$. This gives a solution of mean curvature flow which is written as a graph over $\partial B_{a} \times \mathbb{R}$ and starts from there. Hence it coincides with $\left(\partial B_{t} \times \mathbb{R}\right)_{t \in[a, a+\tau]}$.

(To see the uniqueness for smooth cylinders write a non-cylindrical solution as a graph over the initial cylinder. By the strong maximum principle the difference to the ordinary cylindrical solution attains no interior maximum. Then translate the non-cylindrical solution along the cylinder and again use interior estimates to find convergence to a new solution, and do the translation in such a way that the difference of the limit to the cylindrical solution attains an interior maximum. This contradicts the strong maximum principle.)

Thus, we have shown that the corresponding flows $\left(K_{t}^{R}\right)_{t \in[a, a+\tau]}$ of the open sets starting from $K^{R}$ satisfy

$$
\bigcup_{R>1} K_{t}^{R}=B_{t} \times \mathbb{R}, \quad \text { for } t \in[a, a+\tau] .
$$

Let $\left(W_{t}\right)_{t \in[a, b]}$ be the smallest weak solution in $\mathbb{R}^{n+1}$ with $W_{a}=B_{a} \times \mathbb{R}$. The argument above has shown $W_{t}=B_{t} \times \mathbb{R}$ for $t \in[a, a+\tau]$. The same argument shows, that the maximal time-interval on which $\left(W_{t}\right)$ and $\left(B_{t} \times \mathbb{R}\right)$ coincide is open. It is easy to see that this maximal interval is also closed: Suppose $W_{t}=B_{t} \times \mathbb{R}$ for $t<t_{0}$. Since $\left(W_{t}\right)$ is the smallest solution $W_{t_{0}} \subset B_{t_{0}} \times \mathbb{R}$. To show the reverse inclusion let $x \in B_{t_{0}} \times \mathbb{R}$. Since $\bigcup_{t \in(a, b)} B_{t} \times \mathbb{R} \times\{t\}$ is open we find a ball-solution of mean curvature flow centred at $x$ that is contained in $\left(B_{t} \times \mathbb{R}\right)_{t}$ and contains $\left(x, t_{0}\right)$. This shows $x \in W_{t_{0}}$ since $W_{t}=B_{t} \times \mathbb{R}$ for $t<t_{0}$ and $W_{t}$ is a supersolution and hence contains the ball-solution.

Summarizing we find $W_{t}=B_{t} \times \mathbb{R}$ for all $t \in[a, b]$, i. e. the smallest weak solution starting from $B_{a} \times \mathbb{R}$ is $\left(B_{t} \times \mathbb{R}\right)_{t}$. As $A_{t} \times \mathbb{R}$ is a supersolution with $B_{a} \times \mathbb{R} \subset A_{a} \times \mathbb{R}$ we conclude $B_{b} \times \mathbb{R} \subset A_{b} \times \mathbb{R}$

A shadowflow is a weak solution:

Theorem 3.5. Let $u: \bar{\Omega} \times[0, \infty) \rightarrow[-\infty, \infty]$ be continuous and smooth in the set $\{(x, t) \in \Omega \times(0, \infty):|u(x, t)|<\infty\}$ and suppose $u$ satisfies (GMCF in this set. 
Define $A_{t}$ to be the projection of graph $u(\cdot, t) \cap \mathbb{R}^{n+1}$ onto $\bar{\Omega}$, that is

$$
A_{t}=\{x \in \bar{\Omega}:|u(\cdot, t)|<\infty\} .
$$

Then $\left(A_{t}\right)_{t \in[0, \infty)}$ is a weak solution of mean curvature flow with boundary values $(\{x \in \partial \Omega:|u(x, t)|<\infty\})_{t \in[0, \infty)}$.

Proof. By Lemma 3.4 it suffices to show that $A_{t} \times \mathbb{R}$ is a weak solution.

First we prove that $\left(A_{t} \times \mathbb{R}\right)_{t}$ is a subsolution, which is slightly easier to see. So let $\left(B_{t}\right)_{t \in[a, b]}$ be a family of open bounded subsets of $\mathbb{R}^{n+1}$ such that the boundaries $\left(\partial B_{t}\right)_{t \in[a, b]}$ form a smooth solution of mean curvature flow. Suppose $\overline{B_{a}} \subset(\Omega \backslash$ $\left.\overline{A_{a}}\right) \times \mathbb{R}$. Assume for contradiction that there is $X \in B_{b}$ such that $X \notin\left(\Omega \backslash \overline{A_{b}}\right) \times \mathbb{R}$. By openness of $B_{b}$ we may assume $X \in A_{b} \times \mathbb{R}$. Taking a vertical translation of $\left(B_{t}\right)$ we may further assume $X \in \operatorname{graph} u(\cdot, b)$. This contradicts the avoidance principle.

Now to see that $\left(A_{t} \times \mathbb{R}\right)_{t}$ is a supersolution, suppose $\overline{B_{a}} \subset A_{a} \times \mathbb{R}$ and assume for contradiction that there is $X \in B_{b}$ such that $X \notin A_{b} \times \mathbb{R}$. W.l.o.g. we assume $u\left(X_{1}, \ldots, X_{n}, b\right)=+\infty$ and taking a vertical translation of $\left(B_{t}\right)$ we may assume $u\left(Y_{1}, \ldots, Y_{n}, a\right)<Y_{n+1}$ for all $Y \in \overline{B_{a}}$, i. e. $\overline{B_{a}}$ is above graph $u(\cdot, a)$. But then by the avoidance principle $\overline{B_{b}}$ would be above graph $u(\cdot, b)$ which leads to a contradiction.

\section{Smoothing intersections While Respecting CURVATURE CONDitions}

We observe the following

Lemma 4.1. Let $\Gamma \subset \mathbb{R}^{n}$ be an open or closed, symmetric and convex cone which contains the positive cone. Then the set of real symmetric $n \times n$-matrices with eigenvalues in $\Gamma$ is convex.

Proof. Let $\Gamma$ be open. (The case of closed $\Gamma$ is handled similar.) Let $f: \mathbb{R}^{n} \rightarrow \mathbb{R}$ be the signed distance function to $\partial \Gamma$ (which we assume to be nonempty) such that $\lambda \in \Gamma \Longleftrightarrow f(\lambda)>0$. By the convexity of $\Gamma, f$ is concave. By the symmetry of $\Gamma$, $f$ is symmetric. And because $\Gamma$ contains the positive cone $f$ is increasing in each component of its argument. Then $F(A):=f(\lambda(A))$ is a concave function on the set of symmetric matrices, where $\lambda(A)$ denotes the eigenvalues of $A$ (see e.g. [2, end of $\S 3]$ ). Thus $\{A: F(A)>0\}$ is a convex subset of the symmetric matrices, which finishes the proof.

Proof of Theorem 1.2. The idea is to use distance functions and take a mollified version of $\min$ (denoted $\widetilde{\min }$ ) and to define

$$
\Omega:=\left\{\widetilde{\min }\left(\operatorname{dist}_{\partial A}, \operatorname{dist}_{\partial B}\right)>0\right\}
$$

though we will not directly use the distance functions.

1. Altered distance functions and reference neighbourhoods. First note that since $A \cap B$ is bounded it suffices to consider everything in a large ball. Let $d_{A} \in C^{\infty}\left(\mathbb{R}^{n}\right)$ be such that $d_{A}<0$ in $\mathbb{R}^{n} \backslash \bar{A}$ and in our large ball we have $d_{A}>0$ in $A$ and $d_{A}$ coincides with the signed distance function in a tubular neighbourhood of $\partial A$. Let $g \in C^{\infty}(\mathbb{R})$ be such that $g(0)=0,1 \leq g^{\prime} \leq 2$ and $g^{\prime \prime}(s) \leq-C$ for $|s|<\varepsilon(C)$, where we choose $C>0$ later, and set $a:=g \circ d_{A}$. We derive

$$
\begin{aligned}
D a & =g^{\prime}\left(d_{A}\right) D d_{A}, \\
D^{2} a & =g^{\prime \prime}\left(d_{A}\right) D d_{A} \otimes D d_{A}+g^{\prime}\left(d_{A}\right) D^{2} d_{A} .
\end{aligned}
$$

In a tubular neighbourhood (in our large ball) $D a$ is an eigenvector of $D^{2} a$ with eigenvalue $g^{\prime \prime}\left(d_{A}\right)$. The remaining eigenvalues are $g^{\prime}\left(d_{A}\right)$ times the eigenvalues of $D^{2} d_{A}$ which are

$$
\frac{-\kappa_{i} \circ \pi}{1-d_{A} \cdot \kappa_{i} \circ \pi} \quad(i=1, \ldots, n-1)
$$


Here $\kappa_{i}$ are the principal curvatures at the boundary and $\pi$ denotes the closest point projection onto the boundary. Note that inside $\bar{A}$ these eigenvalues of $D^{2} d_{A}$ are not greater than the negated principle curvatures of the boundary. Because $\Gamma$ contains the positive cone, we find the negation of the eigenvalues of $D^{2} a$ which correspond to eigenvectors orthogonal to $D a$ lie in $\Gamma$.

Now choose $C>0$ from above such that the eigenvalue of $D^{2} a$ which corresponds to the eigenvector $D a$ is the smallest (largest in absolute value) eigenvalue of $D^{2} a$ in a neighbourhood of $\partial A$ (still restricted to a large ball). We refer to this neighbourhood restricted to $\bar{A}$ as the reference neighbourhood of $\partial A$ (in our large ball). In the reference neighbourhood the negations of the eigenvalues of $\left.D^{2} a\right|_{V}$ are in $\Gamma$ for any $(n-1)$-dimensional hyperplane $V$.

Analogously we define $b$ with respect to $\partial B$ and the reference neighbourhood of $\partial B$.

2. Construction. Let $f \in C^{\infty}(\mathbb{R})$ be a function with the following properties

(i) $\min \{s, 0\}-1<f(s)<\min \{s, 0\}$ for $|s|<1$,

(ii) $f(s)=\min \{s, 0\}$ for $|s| \geq 1$,

(iii) $0 \leq f^{\prime} \leq 1$,

(iv) $f^{\prime \prime} \leq 0$.

Define

$$
\begin{aligned}
\Phi:(0,1) \times \mathbb{R}^{n} & \rightarrow \mathbb{R}, \\
(\delta, x) & \mapsto \delta f\left(\frac{a(x)-b(x)}{\delta}\right)+b(x) .
\end{aligned}
$$

This is a mollified version of $\min (a, b)$ with parameter $\delta$. In fact

$$
\min \{a(x), b(x)\}-\delta<\Phi(\delta, x) \leq \min \{a(x), b(x)\}
$$

for all $x \in \mathbb{R}^{n}$ and $\delta \in(0,1)$. We will choose $\Omega$ from the assertion of the form

$$
\Omega_{\delta}:=\left\{x \in \mathbb{R}^{n}: \Phi(\delta, x)>0\right\}
$$

for an appropriate choice of $\delta$

3. Inclusions. Because of $A \cap B=\{\min (a, b)>0\}$ it is obvious from 4.2 ) that $\Omega_{\delta} \subset A \cap B$ holds for all $\delta \in(0,1)$. To check the other inclusion let $x \in$ $A \cap B \backslash(\partial A \cap \partial B)_{\varepsilon}$. By continuity there is $0<\delta_{1}=\delta_{1}(\varepsilon)$ independent of $x$, such that

$$
\max \{a(x), b(x)\}>\delta_{1} .
$$

Now choose $\delta \leq \delta_{1} / 2$ and distinguish two cases: Suppose $|a(x)-b(x)|>\delta$. Then by property (ii) of $f$ we find

$$
\Phi(\delta, x)=\min \{a(x), b(x)\}>0 .
$$

If on the other hand $|a(x)-b(x)| \leq \delta$ then by (4.2), 4.3), and $\delta \leq \delta_{1} / 2$

$$
\Phi(\delta, x)>\min \{a(x), b(x)\}-\delta \geq \delta_{1}-2 \delta \geq 0 .
$$

In summary we find the claimed inclusions, provided that $\delta$ is sufficiently small.

4. Smoothness. We compute

$$
D \Phi=\left(f\left(\frac{a-b}{\delta}\right)-f^{\prime}\left(\frac{a-b}{\delta}\right) \frac{a-b}{\delta}, f^{\prime}\left(\frac{a-b}{\delta}\right)(D a-D b)+D b\right) .
$$

Assuming $\Phi=0$ implies

$$
f\left(\frac{a-b}{\delta}\right)=-\frac{b}{\delta}
$$


and therefore

$$
\partial_{\delta} \Phi=0 \Longleftrightarrow b+\underbrace{f^{\prime}\left(\frac{a-b}{\delta}\right)}_{\in[0,1]}(a-b)=0 .
$$

This occurs only if one of the following is true (note that $\Phi=0$ already implies $a, b \geq 0$, by (4.2)

(I) $a=0$ and $b=0$

(II) $a=0$ and $f^{\prime}\left(\frac{a-b}{\delta}\right)=1$

(III) $b=0$ and $f^{\prime}\left(\frac{a-b}{\delta}\right)=0$.

(I) implies $\Phi \neq 0$, a contradiction. In case (II) we find $\partial_{x} \Phi=D a \neq 0$, because $D a \neq 0$ on $\partial A$, which is where $a=0$ holds. Case (III) is treated analogously to case (II).

Summarizing we obtain $D \Phi \neq 0$ where $\Phi=0$. The implicit function theorem shows, that $\Phi^{-1}(0)$ is a smooth $n$-dimensional submanifold of $(0,1) \times \mathbb{R}^{n}$ with normal $\frac{D \Phi}{|D \Phi|}$.

By applying Sard's theorem to the mapping $\Phi^{-1}(0) \rightarrow(0,1),(\delta, x) \mapsto \delta$ one can show, that for almost all $\delta \in(0,1), \partial_{x} \Phi(\delta, \cdot) \neq 0$ where $\Phi(\delta, \cdot)=0$, so that by the implicit function theorem again $\Omega_{\delta}$ is smooth for almost all $\delta \in(0,1)$ and $\partial \Omega_{\delta}=\left\{x \in \mathbb{R}^{n}: \Phi(\delta, x)=0\right\}$.

We choose $\Omega:=\Omega_{\delta_{0}}$ for such an $\delta_{0} \in(0,1)$ sufficiently small, that the asserted inclusions hold.

5. Curvature condition. Let $x_{0} \in \partial \Omega$ and assume without loss of generality $x_{0}=0$ and further assume that the tangent space of $\partial \Omega$ at $x_{0}=0$ is orthogonal to $e_{n}$. We identify the tangent space and $\mathbb{R}^{n-1} \equiv\left\{\left(\hat{x}, x^{n}\right) \in \mathbb{R}^{n}: x^{n}=0\right\}$. Locally $\partial \Omega$ is a graph over the tangent space at $x_{0}$ : Let $w \in C^{\infty}\left(\mathbb{R}^{n-1}\right)$ and $r>0$ with

$$
B_{r}(0) \cap \Omega=B_{r}(0) \cap\left\{\left(\hat{x}, x^{n}\right) \in \mathbb{R}^{n}: w(\hat{x})<x^{n}\right\} .
$$

We write $\Phi_{\delta_{0}} \equiv \Phi\left(\delta_{0}, \cdot\right)$ and $\hat{D}$ for differentiation with respect to the first $n-1$ components.

The following holds in a neighbourhood of $0 \in \mathbb{R}^{n-1}$ :

$$
\begin{aligned}
\Phi_{\delta_{0}}(\hat{x}, w(\hat{x})) & =0, \\
\hat{D} w(0) & =0, \\
\partial_{n} \Phi_{\delta_{0}}(0) & =\left|D \Phi_{\delta_{0}}(0)\right| .
\end{aligned}
$$

Differentiating (4.4) twice and using (4.5) and 4.6 we obtain

$$
\hat{D}^{2} w(0)=-\frac{\hat{D}^{2} \Phi_{\delta_{0}}(0)}{\left|D \Phi_{\delta_{0}}(0)\right|}
$$

Note that the eigenvalues of $\hat{D}^{2} w(0)$ are the principle curvatures of $\partial \Omega$ at $x_{0}$. Hence, it remains to prove that the negated eigenvalues of $\hat{D}^{2} \Phi_{\delta_{0}}(0)$ lie in $\Gamma$.

We compute

$$
\begin{aligned}
\hat{D}^{2} \Phi_{\delta_{0}}= & \frac{1}{\delta_{0}} f^{\prime \prime}\left(\frac{a-b}{\delta_{0}}\right)(\hat{D} a-\hat{D} b) \otimes(\hat{D} a-\hat{D} b) \\
& +f^{\prime}\left(\frac{a-b}{\delta_{0}}\right)\left(\hat{D}^{2} a-\hat{D}^{2} b\right)+\hat{D}^{2} b .
\end{aligned}
$$

The matrix $(\hat{D} a-\hat{D} b) \otimes(\hat{D} a-\hat{D} b)$ is positive semi-definite and $f^{\prime \prime} \leq 0$ (property (iv)). Since $\Gamma$ contains the positive cone it suffices to consider the second term in 4.7). We distinguish three cases, below. But first we observe the following: We may assume that $\varepsilon>0$ is so small that $(\partial A \cap \partial B)_{\varepsilon} \cap(A \cap B)$ is contained in the 
intersection of the reference neighbourhoods of $\partial A$ and $\partial B$. Then $x_{0}=0 \in \partial \Omega$ is in $\partial A$ or $\partial B$ or in the reference neighbourhoods of both $\partial A$ and $\partial B$.

(I) $a(0)-b(0) \geq \delta_{0}:$ Then

$$
f^{\prime}\left(\frac{a(0)-b(0)}{\delta_{0}}\right)=0
$$

The term in question becomes $\hat{D}^{2} b$. Moreover, in this case $0=\Phi_{\delta_{0}}(0)=b(0)$ holds. That is why we are in the reference neighbourhood of $\partial B$, where the negations of the eigenvalues of $\hat{D}^{2} b$ lie in $\Gamma$.

(II) $a(0)-b(0) \leq-\delta_{0}$ : This case is treated similiar.

(III) $|a(0)-b(0)|<\delta_{0}$ : Here, by property (i) of $f, 0 \notin \partial A, \partial B$. Therefore 0 must be in the reference neighbourhoods of $\partial A$ and $\partial B$. As a convex combination of two matrices, whose negated eigenvalues lie in $\Gamma$, the negated eigenvalues of

$$
f^{\prime}\left(\frac{a-b}{\delta_{0}}\right)\left(\hat{D}^{2} a-\hat{D}^{2} b\right)+\hat{D}^{2} b
$$

the matrix in question, are in $\Gamma$ by Lemma 4.1

In any case, the principal curvatures of $\partial \Omega$ at the point $x_{0}=0$ lie in $\Gamma$.

\section{REFERENCES}

1. G. Bellettini, M. Novaga. Comparison results between minimal barriers and viscosity solutions for geometric evolutions. Annali della Scuola Normale Superiore di Pisa - Classe di Scienze 26.1 (1998): 97-131.

2. L. Caffarelli, L. Nirenberg, J. Spruck, The Dirichlet Problem for nonlinear second order elliptic equations, III: Function of the eigenvalues of the Hessian, Acta Math. 155 (1985), 261-301.

3. K. Choi, P. Daskalopoulos, The $Q_{k}$ flow on complete non-compact graphs, arXiv:1603.03453 1

4. K. Choi, P. Daskalopoulos, L. Kim, K. Lee, The evolution of complete non-compact graphs by powers of Gauss curvature, arXiv:1603.04286v1

5. K. Ecker and G. Huisken, Interior estimates for hypersurfaces moving by mean curvature, Invent. Math. 105 (1991), no. 3, 547-569.

6. E. Giusti, Direct Methods in the Calculus of Variations, World Scientific Publishing Co. Ptc. Ltd., 2003, ISBN 981-238-043-4

7. G. Huisken, Non-parametric Mean Curvature Evolution with Boundary Conditions, Journal of Differential Equations 77, 369-378 (1989)

8. T. Ilmanen, The Level-set Flow on a Manifold, Proc. Symp. Pure Math. 54 Pt. 1 (1993) 193204.

9. M. Sáez Trumper and O. Schnürer, Mean curvature flow without singularities, J. Differential Geom. 97 (2014), no. 3, 545-570.

10. B. White, The Topology of Hypersurfaces Moving by Mean Curvature, Communication in Analysis and Geometry Vol. 3, Number 2, 317-333, 1995.

Wolfgang Maurer, Fachbereich Mathematik und Statistik, Universität Konstanz, 78457 Konstanz, Germany 